\title{
EXPONENTIAL CONVERGENCE FOR A CONVEXIFYING EQUATION
}

\author{
${\text { Guillaume } \text { Carlier }^{1} \text { And Alfred Galichon }}^{2}$
}

\begin{abstract}
We consider an evolution equation similar to that introduced by Vese in [Comm. Partial Diff. Eq. 24 (1999) 1573-1591] and whose solution converges in large time to the convex envelope of the initial datum. We give a stochastic control representation for the solution from which we deduce, under quite general assumptions that the convergence in the Lipschitz norm is in fact exponential in time.
\end{abstract}

Mathematics Subject Classification. 35B40, 49L20, 93E20.

Received November 20, 2010.

Published online July 22, 2011.

\section{INTRODUCTION}

In an interesting paper [12], Vese considered the following PDE:

$$
\partial_{t} u=\sqrt{1+|\nabla u|^{2}} \min \left(0, \lambda_{1}\left(D^{2} u\right)\right),\left.u\right|_{t=0}=u_{0}
$$

where $\lambda_{1}\left(D^{2} u\right)$ denotes the smallest eigenvalue of the Hessian matrix $D^{2} u$. Vese proved, under quite general assumptions on the initial condition $u_{0}$, that the viscosity solution of (1.1) converges as $t \rightarrow \infty$ to $u_{0}^{* *}$ the convex envelope of $u_{0}$. Starting from this result, Vese developed an original and purely PDE approach to approximate convex envelopes (which is in general a delicate problem as soon as the space dimension is larger than 2). More recently, Oberman [7-9], noticed that the convex envelope can be directly characterized via a nonlinear elliptic PDE of obstacle type and developed this idea for numerical computation of convex envelopes as well. As noticed by Oberman, the solution of the PDE he introduced naturally has a stochastic control representation. This is of course also the case for the evolutionary equation and, as we shall see, this representation will turn out to be very useful to obtain convergence estimates.

In the present paper, we will focus on an evolution equation similar to (1.1) and will study some of its properties thanks to the stochastic control representation of the solution.

\footnotetext{
Keywords and phrases. Convex envelope, viscosity solutions, stochastic control representation, nonautonomous gradient flows.

${ }^{1}$ CEREMADE, UMR CNRS 7534, Université Paris IX Dauphine, Pl. de Lattre de Tassigny, 75775 Paris Cedex 16, France. carlier@ceremade.dauphine.fr

2 Département d'Économie, UMR CNRS 7176, École polytechnique, 91128 Palaiseau Cedex, France.

alfred.galichon@polytechnique.edu
} 
The paper is organized as follows. In Section 2, we introduce the convexifying evolution equation and recall some basic facts about convex envelopes. In Section 3, we give a stochastic control representation for the solution of the convexifying evolution equation. Section 4 gives some regularity properties of the solution. Our exponential convergence result is then proved in Section 5 by simple probabilistic arguments. Finally, Section 6 is devoted to some concluding remarks. We apply our exponential convergence result to study the behavior of some nonautonomous gradient flow and we finally make a link with some geometric flow by considering a family of interpolating problems.

\section{A CONVEXIFYing EVOLUTION EQUATION}

In the present paper, we will consider a slight variant of (1.1), namely:

$$
\partial_{t} u(t, x)=\min \left(0, \lambda_{1}\left(D^{2} u(t, x)\right)\right),(t, x) \in(0 ; \infty) \times \mathbb{R}^{d},\left.u\right|_{t=0}=u_{0} .
$$

From a PDE point of view, it may be worthwile to observe that the nonlinear operator involved in the equation may be considered, in a degenerate case, as one of the Pucci extremal operators, which have been extensively studied in the literature on fully nonlinear PDE's (see for instance the book by Caffarelli and Cabré [2]).

In the sequel, we shall refer to (2.1) as the convexifying evolution equation. Following the same arguments of the proof of Vese [12] (also see Rem. 3.4 below), one can prove under mild assumptions on $u_{0}$ that the solution converges pointwise to the convex envelope $u_{0}^{* *}$ of the initial condition. Our aim will be to quantify this convergence and this goal will be achieved rather easily by using a stochastic representation formula for the solution of (2.1). Before we do so, let us recall some basic facts about the convex envelope.

Given a continuous (say) and bounded from below function $u_{0}$ defined on $\mathbb{R}^{d}$, the convex envelope of $u_{0}^{* *}$ is the largest convex function that is everywhere below $u_{0}$. The convex envelope is a very natural object in many contexts and in particular in optimization since $u_{0}$ and $u_{0}^{* *}$ have the same infimum but $u_{0}^{* *}$ is in principle much simpler to minimize since it is convex. One can also define $u_{0}^{* *}$ as the supremum of all affine functions that are below $u_{0}$ and thus define $u_{0}^{* *}$ as the "Legendre transform of the Legendre transform" of $u_{0}$ (and this is where the notation "**" comes from). Rather than iterating the Legendre transform, let us recall the well-known formula:

$$
u_{0}^{* *}(x)=\inf \left\{\sum_{i=1}^{d+1} \lambda_{i} u_{0}\left(x_{i}\right): \lambda_{i} \geq 0, \sum_{i=1}^{d+1} \lambda_{i}=1, \sum_{i=1}^{d+1} \lambda_{i} x_{i}=x\right\}, \forall x \in \mathbb{R}^{d}
$$

(the fact that one can restrict to $d+1$ points follows from Carathéodory's theorem) which can also be written in probalistic terms as

$$
u_{0}^{* *}(x)=\inf \left\{\mathbb{E}\left(u_{0}(x+X)\right): \mathbb{E}(X)=0\right\} .
$$

The latter formula strongly suggests that a good approximation for the convex envelope should be

$$
u(t, x):=\inf _{\sigma:|\sigma| \leq 1}\left\{\mathbb{E}\left(u_{0}\left(x+\int_{0}^{t} \sqrt{2} \sigma_{s} \mathrm{~d} W_{s}\right)\right)\right\}
$$

for large $t$ where $\left(W_{s}\right)_{s \geq 0}$ is a standard Brownian motion, $\sigma_{s}$ is a $d \times d$-matrix valued process that is adapted to the Brownian filtration and $|\sigma|$ stands for the matrix norm $|\sigma|:=\sqrt{\operatorname{Tr}\left(\sigma \sigma^{T}\right)}$.

In order to keep things as elementary as possible, from now on, we shall always assume that $u_{0}$ satisfies:

$$
u_{0} \in C^{1,1}\left(\mathbb{R}^{d}\right), \lim _{|x| \rightarrow \infty} \frac{u_{0}(x)}{|x|}=+\infty, \exists R_{0}>0: u_{0}=u_{0}^{* *} \text { outside } \bar{B}_{R_{0}} .
$$

The coercivity assumption guarantees that the infimum in formula (2.2) is actually achieved. The assumption that $u_{0}$ and $u_{0}^{* *}$ agree outside of some ball, will be convenient and allow us to work mainly on a ball instead 
of on the whole space. Indeed, thanks to (2.5), we claim that there exists $R \geq R_{0}$ such that, for any $x \in \bar{B}_{R_{0}}$, in the formula $(2.2)$, it is enough to restrict the minimization to points $x_{i}$ in $\bar{B}_{R}$ :

$$
u_{0}^{* *}(x)=\inf \left\{\sum_{i=1}^{d+1} \lambda_{i} u_{0}\left(x_{i}\right): \lambda_{i} \geq 0, \sum_{i=1}^{d+1} \lambda_{i}=1, \sum_{i=1}^{d+1} \lambda_{i} x_{i}=x, x_{i} \in \bar{B}_{R}\right\},
$$

for every $x \in \bar{B}_{R_{0}}$. To prove this claim, it is enough to remark that whenever the $\lambda_{i}>0$ 's and $x_{i}$ 's solve (2.2) then $u_{0}^{* *}$ is actually affine on the convex hull of the points $x_{i}$, and then to note that the sets on which $u_{0}^{* *}$ is affine are necessarily bounded thanks to the coercivity of $u_{0}$ and the fact that $u_{0}=u_{0}^{* *}$ outside $\bar{B}_{R_{0}}$. Finally, the assumption that $u_{0}$ is $C^{1,1}$ implies that so is $u_{0}^{* *}$ (see [6]) and we will see that it also implies that $u(t,$. ) remains $C^{1,1}$.

\section{Stochastic CONTROL REPRESENTATION}

As we shall see (but this should already be clear to stochastic control-oriented readers), the value function of (2.4) is in fact characterized by the PDE:

$$
\partial_{t} v=\min \left(0, \lambda_{1}\left(D^{2} v\right)\right)
$$

in the viscosity sense (see [4] for an overview of the theory of viscosity solutions) that we now recall (for the sake of simplicity, we will restrict ourselves to the framework of continuous solutions which is sufficient in our context):

Definition 3.1. Let $\Omega$ be some open subset of $\mathbb{R}^{d}$ and let $v$ be continuous on $(0,+\infty) \times \Omega$, then $v$ is:

- a viscosity subsolution of $(3.1)$ on $(0,+\infty) \times \Omega$ if for every smooth function $\varphi \in C^{2}((0,+\infty) \times \Omega)$ and every $\left(t_{0}, x_{0}\right) \in(0,+\infty) \times \Omega$ such that $(v-\varphi)\left(t_{0}, x_{0}\right)=\max _{(0,+\infty) \times \Omega}(v-\varphi)$ one has

$$
\partial_{t} \varphi\left(t_{0}, x_{0}\right) \leq \min \left(0, \lambda_{1}\left(D^{2} \varphi\left(t_{0}, x_{0}\right)\right)\right)
$$

- a viscosity supersolution of $(3.1)$ on $(0,+\infty) \times \Omega$ if for every smooth function $\varphi \in C^{2}((0,+\infty) \times \Omega)$ and every $\left(t_{0}, x_{0}\right) \in(0,+\infty) \times \Omega$ such that $(v-\varphi)\left(t_{0}, x_{0}\right)=\min _{(0,+\infty) \times \Omega}(v-\varphi)$ one has

$$
\partial_{t} \varphi\left(t_{0}, x_{0}\right) \geq \min \left(0, \lambda_{1}\left(D^{2} \varphi\left(t_{0}, x_{0}\right)\right)\right)
$$

- a viscosity solution of $(3.1)$ on $(0,+\infty) \times \Omega$ if it is both a viscosity subsolution and a viscosity supersolution.

We then have the following stochastic representation formula for (2.1):

Theorem 3.2. Under assumption (2.5), there is a unique continuous function $u$ on $[0,+\infty) \times \mathbb{R}^{d}$ that agrees with $u_{0}$ at $t=0$, that is a viscosity solution of (2.1) and that agrees with $u_{0}^{* *}$ outside $B_{R_{0}}$. It admits the following representation

$$
u(t, x)=\inf _{\sigma:|\sigma| \leq 1}\left\{\mathbb{E}\left(u_{0}\left(x+\int_{0}^{t} \sqrt{2} \sigma_{s} \mathrm{~d} W_{s}\right)\right)\right\}, t \geq 0, x \in \mathbb{R}^{d}
$$

where $\left(W_{s}\right)_{s \geq 0}$ is a standard Brownian motion and $|\sigma|$ stands for the matrix norm $|\sigma|:=\sqrt{\operatorname{Tr}\left(\sigma \sigma^{T}\right)}$.

Proof. Recalling that for every symmetric matrix $S$ one has

$$
\min \left(0, \lambda_{1}(S)\right)=\min _{|\sigma| \leq 1} \operatorname{Tr}\left(\sigma \sigma^{T} S\right)
$$

the fact that formula (3.2) actually defines a viscosity solution of (2.1) is a classical fact from stochastic control theory (see for instance [5] or [11]), assumption (2.5) guarantees that the function defined by (3.2) agrees with 
$u_{0}^{* *}$ outside $B_{R_{0}}$. Continuity (local Lipschitz continuity in fact) of the value function defined by (3.2) will be established in Section 4. It follows from well-known parabolic comparison principles (e.g Thm. 4.1 in [3]) that if $T>0, v_{1}$ (respectively $v_{2}$ ) is a viscosity subsolution (respectively supersolution) of $(2.1)$ on $(0, T) \times B_{R_{0}}$ such that $v_{1}(t, x) \leq v_{2}(t, x)$ for $(t, x) \in\{0\} \times B_{R_{0}} \cup[0, T] \times \partial B_{R_{0}}$ then $v_{1} \leq v_{2}$ on $[0, T] \times B_{R_{0}}$. This proves the uniqueness of a viscosity solution of (2.1) which equals $u_{0}$ at $t=0$ and that agrees with $u_{0}^{* *}$ outside $B_{R_{0}}$.

Remark 3.3 (optimal feedback control). Very formally, if the solution $u$ of the PDE were very well-behaved then, as usual in control theory, one could find an optimal feedback (Markov) control depending on $D^{2} u$ (since there is no drift). Introduce a time-dependent vector field $Z=Z(t, y)$, as follows. If $\lambda_{1}\left(D^{2} u(t, y)\right)<0$, then let $Z(t, y)$ be a unit eigenvector associated to $\lambda_{1}\left(D^{2} u(t, y)\right)$ and let $Z(t, y)=0$ otherwise. So that in any case:

$$
\operatorname{Tr}\left(\sigma(t, y) \sigma(t, y)^{T} D^{2} u(t, y)\right)=\min \left(0, \lambda_{1}\left(D^{2} u(t, y)\right)\right), \text { and }|\sigma(t, y)| \leq 1,
$$

where $\sigma$ is the projector

$$
\sigma(t, y):=Z(t, y) \otimes Z(t, y)
$$

Of course the problem is that $\sigma$ is not well-defined: not only $u$ does not need to be $C^{2}$ but also it may be the case that $\lambda_{1}<0$ has multiplicity larger than 2. Ignoring those serious issues, let us consider the SDE:

$$
\mathrm{d} Y_{t}=\sqrt{2} \sigma\left(t, Y_{t}\right) \mathrm{d} W_{t}
$$

then $\sigma$ is (again very formally) an optimal feedback control. We then have for $t>s \geq 0$

$$
u(t, y)=\mathbb{E}\left[u\left(s, Y_{t}\right) \mid Y_{s}=y\right],
$$

and, formally, the envelope theorem gives

$$
\nabla u(t, y)=\mathbb{E}\left[\nabla u\left(s, Y_{t}\right) \mid Y_{s}=y\right] .
$$

Finally, notice that the drift of $u\left(t, Y_{t}\right)$ is the nonpositive quantity given by

$$
\partial_{t} u\left(t, Y_{t}\right)+\operatorname{Tr}\left(\sigma \sigma^{T} D^{2} u\left(t, Y_{t}\right)\right)=2 \min \left(0, \lambda_{1}\left(D^{2} u\left(t, Y_{t}\right)\right)\right) .
$$

Remark 3.4. One has $u_{0}^{* *} \leq u(t,.) \leq u_{0}$ and $u(., x)$ is nonincreasing and thus monotonically converges to $v(x):=\lim _{t \rightarrow \infty} u(t, x)=\inf _{t>0} u(t, x)$. Now, as shown by Vese in [12], $v$ is necessarily convex (it is a viscosity solution of the stationary equation) and since $u_{0}^{* *} \leq u(t,.) \leq u_{0}$ this gives $v=u_{0}^{* *}$. In other words, $u$ pointwise monotonically converges to the convex envelope of the initial condition. Of course, in view of the representation formulas (3.2) and (2.2) this convergence is not surprising. We shall see in the next sections how (3.2) can easily give much more precise informations and provide in a simple way very strong convergence estimates.

\section{REgulaRity PROPERTIES OF $u$}

Lemma 4.1. If $M>0$ is such that $u_{0}-\frac{M}{2}|\cdot|^{2}$ is concave then $u(t,)-.\frac{M}{2}|\cdot|^{2}$ is concave for every $t>0$.

Proof. Set $v_{0}:=u_{0}-\frac{M}{2}|.|^{2}$ and let $\left(X_{\alpha}\right)_{\alpha \in A}$ be a family of centered, $\mathbb{R}^{d}$-valued, square integrable random variables, then define

we then have

$$
\varphi(x)=\inf _{\alpha \in A} \mathbb{E}\left(u_{0}\left(x+X_{\alpha}\right)\right), x \in \mathbb{R}^{d}
$$

$$
\varphi(x)-\frac{M}{2}|x|^{2}=\inf _{\alpha \in A}\left\{\mathbb{E}\left(v_{0}\left(x+X_{\alpha}\right)+\frac{M}{2}\left|X_{\alpha}\right|^{2}\right)\right\}
$$

so that $\varphi-\frac{M}{2}|.|^{2}$ is concave as an infimum of concave functions. This proves the desired claim. 
Proposition 4.2. Let $u_{0}$ be $C^{1,1}$ and let $M:=\left\|D^{2} u_{0}\right\|_{\infty}$, then for every $(t, s) \in(0,+\infty)$ and every $x \in \mathbb{R}^{d}$ one has

$$
|u(t, x)-u(s, x)| \leq M|s-t|
$$

and $u(t,$.$) is C^{1,1}$ for every $t$ and more precisely, one has $\left\|D^{2} u(t, .)\right\|_{\infty} \leq M$.

Proof. Let $0<t<s$, we already know that $u(t,.) \geq u(s,$.$) . Let us assume for a moment that u_{0}$ is smooth and let $x \in \mathbb{R}^{d}$ and let $\sigma$ be an adapted process with values in the set of matrices with norm less than 1 such that

$$
\mathbb{E}\left(u_{0}\left(x+\int_{0}^{s} \sqrt{2} \sigma \mathrm{d} W\right)\right) \leq u(s, x)+\varepsilon
$$

then defining

$$
Y_{h}:=x+\int_{0}^{h} \sqrt{2} \sigma \mathrm{d} W, Z_{h}:=u_{0}\left(Y_{h}\right), s \geq h \geq 0
$$

thanks to Itô's formula, we thus get:

$$
\begin{aligned}
u(t, x) & \leq \mathbb{E}\left(Z_{t}\right) \leq u(s, x)+\varepsilon-\mathbb{E}\left(Z_{s}-Z_{t}\right) \\
& =u(s, x)+\varepsilon-\mathbb{E}\left(\int_{t}^{s} \operatorname{Tr}\left(\sigma \sigma^{T} D^{2} u_{0}\left(Y_{h}\right)\right) \mathrm{d} h\right) \\
& \leq u(s, x)+\varepsilon+M(s-t)
\end{aligned}
$$

and we conclude that (4.1) holds by letting $\varepsilon \rightarrow 0^{+}$. In the general case, one applies the same argument to the regularization $\rho_{n} \star u_{0}$ (where $\rho_{n}$ is, as usual, a sequence of mollifyers) and then passes to the limit to obtain (4.1).

Using (2.1), we then quite easily obtain that

$$
\lambda_{1}\left(D^{2} v(t, x)\right) \geq 0 \text { on }(0,+\infty) \times \mathbb{R}^{d}, \text { with } v(t, x):=u(t, x)+\frac{M}{2}|x|^{2}
$$

in the viscosity sense which means that as soon as $\varphi$ is smooth and $v-\varphi$ has a (local or global) maximum at $\left(t_{0}, x_{0}\right) \in(0,+\infty) \times \mathbb{R}^{d}$ then $\lambda_{1}\left(D^{2} \varphi\left(t_{0}, x_{0}\right)\right) \geq 0$. To see that this implies that $v(t,$.$) is convex, we invoke$ the same arguments as in Lemma 1 in [1]. Assume on the contrary that there are $t_{0}>0, x_{0}, y_{0}$ in $\mathbb{R}^{d}$ and $\lambda \in(0,1)$ such that $v\left(t_{0}, \lambda x_{0}+(1-\lambda) y_{0}\right)>\lambda v\left(t_{0}, x_{0}\right)+(1-\lambda) v\left(t_{0}, y_{0}\right)$. Without loss of generality, denoting elements of $\mathbb{R}^{d}$ as $\left(x_{1}, x^{\prime}\right) \in \mathbb{R} \times \mathbb{R}^{d-1}$, we may assume that $y_{0}=(0,0), x_{0}=(1,0)$, we may also assume (adding if necessary some suitable affine function to $v)$ that $v\left(t_{0}, 0\right)=v\left(t_{0},(1,0)\right)<0$ and $v\left(t_{0},(\lambda, 0)\right)>0$. We then choose $h \in\left(0, t_{0}\right)$ and $r>0$ such that

$$
v\left(t,\left(0, x^{\prime}\right)\right)<0, v\left(t,\left(1, x^{\prime}\right)\right)<0, \forall\left(t, x^{\prime}\right) \in\left[t_{0}-h, t_{0}+h\right] \times B_{r} .
$$

We then define

$$
\Omega:=\left\{\left(x_{1}, x^{\prime}\right) \in(0,1) \times B_{r}\right\}, Q:=\left(t_{0}-h, t_{0}+h\right) \times \Omega
$$

and choose $\alpha>0$ such that $v\left(t_{0},(\lambda, 0)\right)>\frac{\alpha \lambda(1-\lambda)}{2}$. We then define

$$
\varphi\left(t,\left(x_{1}, x^{\prime}\right)\right):=\frac{\alpha}{2} x_{1}\left(1-x_{1}\right)+\frac{\beta}{2}\left|x^{\prime}\right|^{2}+\frac{\gamma}{2}\left(t-t_{0}\right)^{2}
$$

with $\beta$ and $\gamma$ chosen so that

$$
\beta r^{2} \geq 2 \max _{\bar{Q}} v, \gamma h^{2} \geq 2 \max _{\bar{Q}} v .
$$

We then have $v\left(t_{0},(\lambda, 0)\right)-\varphi\left(t_{0},(\lambda, 0)\right)>0$ and by $(4.2)-(4.3), v-\varphi \leq 0$ on $\partial Q$, hence $v-\varphi$ achieves its maximum on $\bar{Q}$ at an interior point of $Q$, but at this point one should have $0 \leq \lambda_{1}\left(D^{2} \varphi\right)=-\alpha$ which gives the desired contradiction. This proves that $u(t,)+.\frac{M}{2}|.|^{2}$ is convex for every $t$. Together with Lemma 4.1 this enables us to conclude that $u$ remains semiconvex and semiconcave is hence $C^{1,1}$ with the estimate $\left\|D^{2} u(t, .)\right\|_{\infty} \leq M$. 
Proceeding as in the proof of the two previous results and using the fact that the PDE is autonomous, one gets:

Corollary 4.3. Suppose $u_{0}$ satisfies $(2.5)$, then $\operatorname{Essinf} \lambda_{1}\left(D^{2} u(t,).\right)$ is nondecreasing with respect to $t$ and $\operatorname{Esssup} \lambda_{d}\left(D^{2} u(t,).\right)$ is nonincreasing with respect to $t$ (where $\lambda_{d}$ stands for the largest eigenvalue).

\section{Exponential Convergence to the COnVEX EnVElope}

Before we state our result concerning the convergence of $u(t,$.$) to u_{0}^{* *}$, we need two elementary lemmas.

Lemma 5.1. Let $v: \mathbb{R}^{d} \rightarrow \mathbb{R}$ and $M \geq 0$ be such that $v+\frac{M}{2}||^{2}$ is convex, then for every $r>0$ one has:

$$
\|\nabla v\|_{L^{\infty}\left(B_{r}\right)} \leq 2\left(M\|v\|_{L^{\infty}\left(B_{r+r^{\prime}}\right)}\right)^{1 / 2} \text { with } r^{\prime}=\frac{2}{M r}\|v\|_{L^{\infty}\left(B_{2 r}\right)}+\frac{r}{2}
$$

Proof. Let $r>0, R>0$, for $x \in B_{r}$ a point of differentiability of $v$ (which is a.e. the case) and $h \in B_{R}$ in $\mathbb{R}^{d}$, one first has

$$
2\|v\|_{L^{\infty}\left(B_{r+R}\right)} \geq v(x+h)-v(x) \geq \nabla v(x) \cdot h-\frac{M}{2}|h|^{2} .
$$

Taking $r=R, h=r \nabla v(x)|/| \nabla v(x) \mid$ and maximizing with respect to $x \in B_{r}$ thus gives

$$
\|\nabla v\|_{L^{\infty}\left(B_{r}\right)} \leq \frac{2}{r}\|v\|_{L^{\infty}\left(B_{2 r}\right)}+\frac{M r}{2}
$$

We then take $R=r^{\prime}$ with $r^{\prime}$ defined by (5.1) and set $h=\nabla v(x) / M$, thanks to (5.3), $h \in B_{R}$, using (5.2) again, we then get

which finally gives (5.1).

$$
\frac{|\nabla v(x)|^{2}}{2 M} \leq 2\|v\|_{L^{\infty}\left(B_{r+r^{\prime}}\right)}, \forall x \in B_{r}
$$

Lemma 5.2. Let $\left(B_{t}\right)$ be a standard one-dimensional Brownian motion, let $r>0, x \in(-r, r)$ and

$$
\tau:=\inf \left\{t>0: x+B_{t} \notin[-r, r]\right\}
$$

then for every $t>0$, one has

$$
\mathbb{P}(\tau \geq t) \leq q(r)^{t-1} \text {, with } q(r):=\frac{1}{\sqrt{2 \pi}} \int_{-2 r}^{2 r} \mathrm{e}^{-\frac{s^{2}}{2}} \mathrm{~d} s .
$$

Proof. Let $n$ be the integer part of $t$, we then have

$$
\mathbb{P}(\tau \geq t) \leq \mathbb{P}\left(\left|B_{k}-B_{k-1}\right| \leq 2 r, \text { for } k=1, \ldots, n\right)
$$

and since $\left(B_{k}-B_{k-1}\right)_{k=1, \ldots, n}$ are independent and normally distributed random variables, we immediately get the desired estimate.

Our main result then reads as:

Theorem 5.3. Under assumption (2.5), there exist $C \geq 0$ and $\lambda>0$ such that

$$
\left\|u(t, .)-u_{0}^{* *}\right\|_{L^{\infty}} \leq C \mathrm{e}^{-\lambda t}, \forall t \geq 0
$$

and

$$
\left\|\nabla u(t, .)-\nabla u_{0}^{* *}\right\|_{L^{\infty}} \leq C \mathrm{e}^{-\lambda t}, \quad \forall t \geq 0 .
$$


Proof. First let us remark that if $x \notin \bar{B}_{R_{0}}$, there is nothing to prove. Let us then recall (2.6), thanks to (2.5), there is some ball $\bar{B}_{R}$ containing $\bar{B}_{R_{0}}$, such that for any $x \in \bar{B}_{R_{0}}$, in the formula (2.2), it is enough to restrict the minimization to points $x_{i}$ in $\bar{B}_{R}$. Let then $x \in B_{R_{0}}$, let $\left(x_{1}, \ldots, x_{d+1}\right) \in \bar{B}_{R}^{d+1}$ and $\left(\lambda_{1}, \ldots, \lambda_{d+1}\right)$ be nonnegative such that

$$
\sum_{i=1}^{d+1} \lambda_{i}=1, \sum_{i=1}^{d+1} \lambda_{i} x_{i}=x, \sum_{i=1}^{d+1} \lambda_{i} u_{0}\left(x_{i}\right)=u_{0}^{* *}(x) .
$$

We shall also assume that the points $\left(x_{1}, \ldots, x_{d+1}\right)$ are affinely independent (and this is actually without loss of generality for what follows), the coefficients $\lambda_{i}$ are then uniquely defined and are the unique barycentric coordinates of $x$ in the simplex $K$ which is the convex hull of the points $\left(x_{1}, \ldots, x_{d+1}\right)$. We shall also assume that all the coefficients $\lambda_{i}$ are strictly positive (again this is not a restriction).

Let $\varepsilon>0$ and let $\sigma_{s}=0$, for $s \in[0, \varepsilon]$, then set

$$
v_{1}:=\frac{W_{\varepsilon}}{\left|W_{\varepsilon}\right|}, \tau_{1}:=\inf \left\{t \geq \varepsilon: x+\sqrt{2} v_{1} \otimes v_{1}\left(W_{t}-W_{\varepsilon}\right) \notin K\right\}
$$

and $\sigma_{s}=v_{1} \otimes v_{1}$ for $s \in\left(\varepsilon, \tau_{1}\right]$. By construction, $x+\sqrt{2} v_{1} \otimes v_{1}\left(W_{\tau_{1}}-W_{\varepsilon}\right)$ a.s. belongs to a facet of $K$ of dimension $d-1$. Let us denote by $K_{1}$ this facet and by $E_{1}$ the hyperplane parallel to this facet. Let then $v_{2}$ be $\mathcal{F}_{\varepsilon}$-measurable and uniformly distributed on $S^{d} \cap E_{1}$ and define

$$
\tau_{2}:=\inf \left\{t \geq \tau_{1}: x+\sqrt{2} v_{1} \otimes v_{1}\left(W_{\tau_{1}}-W_{\varepsilon}\right)+\sqrt{2} v_{2} \otimes v_{2}\left(W_{t}-W_{\tau_{1}}\right) \notin K_{1}\right\}
$$

and $\sigma_{s}=v_{2} \otimes v_{2}$ for $x \in\left(\tau_{1}, \tau_{2}\right]$.

We repeat inductively this construction $d$ times and define successive (random and adapted) times $\tau_{k}, k=$ $1, \ldots, d$, directions $v_{1}, \ldots, v_{k}$, and a piecewise constant control $\sigma_{s}=v_{k} \otimes v_{k}$ for $s \in\left(\tau_{k-1}, \tau_{k}\right]$, in such a way that $x+\int_{0}^{t} \sqrt{2} \sigma_{s} \mathrm{~d} W_{s}$ belongs to a facet $K_{k}$ of dimension $d-k$ for $t \in\left[\tau_{k}, \tau_{k+1}\right]$. Let us extend the control $\sigma$ by 0 after time $\tau_{d}$ and set

$$
Y_{t}:=x+\sqrt{2} \int_{0}^{t} \sigma_{s} \mathrm{~d} W_{s}=Y_{t \wedge \tau_{d}}
$$

and remark that at time $\tau_{d}$ the previous process has hit one of the vertices of $K$. By construction $\left(Y_{t}\right)_{t}$ is a continuous martingale and it is bounded since it takes values in the compact $K$, it therefore converges to $Y_{\tau_{d}}$ which is a discrete random variables with values in the vertices of $K,\left\{x_{1}, \ldots, x_{d+1}\right\}$, we then have

$$
\mathbb{E}\left(Y_{\tau_{d}}\right)=x=\sum_{i=1}^{d+1} \mathbb{P}\left(Y_{\tau_{d}}=x_{i}\right) x_{i}
$$

which implies that $\mathbb{P}\left(Y_{\tau_{d}}=x_{i}\right)=\lambda_{i}$ by uniqueness of the barycentric coordinates. We thus have:

$$
u_{0}^{* *}(x)=\mathbb{E}\left(u_{0}\left(Y_{\tau_{d}}\right)\right)
$$

and then using the fact that $Y_{t}$ takes values in $K$ and that $u_{0}$ is locally Lipschitz:

$$
\begin{gathered}
u(t, x) \leq \mathbb{E}\left(u_{0}\left(Y_{t}\right)\right) \leq \mathbb{E}\left(u_{0}\left(Y_{\tau_{d}}\right)\right)+\left\|\nabla u_{0}\right\|_{L^{\infty}(K)} \mathbb{E}\left(\left|Y_{t}-Y_{\tau_{d}}\right|\right) \\
\leq u_{0}^{* *}(x)+\operatorname{diam}(K)\left\|\nabla u_{0}\right\|_{L^{\infty}(K)} \mathbb{P}\left(\tau_{d} \geq t\right)
\end{gathered}
$$

We then remark that

$$
\left\{\tau_{d} \geq t\right\} \subset \bigcup_{k=1}^{d}\left\{T_{k} \geq \frac{t-\varepsilon}{d}\right\}
$$


where the $T_{k}$ 's are the times the process $\left(Y_{s}\right)_{s}$ spends on the (random) facet $K_{k-1}$ (setting $K_{0}=K$ ), the previous probabilities can therefore be estimated by the probability that a one-dimensional Brownian motion spends more than $\frac{(t-\varepsilon)}{d}$ time in the interval $[-\operatorname{diam}(K)$, diam $(K)]$. Using Lemma 5.2, we thus get

$$
\mathbb{P}\left(\tau_{d} \geq t\right) \leq M \mathrm{e}^{-\lambda(t-\varepsilon)}
$$

for constants $M$ and $\lambda>0$ that depend only on $d$ and $\operatorname{diam}(K)$. Letting $\varepsilon \rightarrow 0$, we then obtain

$$
u(t, x) \leq u_{0}^{* *}(x)+\operatorname{diam}(K)\left\|\nabla u_{0}\right\|_{L^{\infty}(K)} M \mathrm{e}^{-\lambda t}
$$

since we already know that $u(t,.) \geq u_{0}^{* *}$, this terminates the proof of (5.4).

Finally, the estimate (5.5) easily follows from (5.4), Lemma 5.1 and the fact that $u(t,)-.u_{0}^{* *}$ remains uniformly semiconcave thanks to Lemma 4.1 .

Remark 5.4. Let us remark that in the inequality (5.4) in Theorem 5.3, the constant $\lambda$ only depends on the dimension and the diameter of the faces of the convex envelope on the set where $\left\{u_{0}>u_{0}^{* *}\right\}$ whereas the constant $C$ also depends on the Lipschitz constant of $u_{0}$ on the set of such faces. In (5.5), $C$ also depends on $\left\|D^{2} u_{0}\right\|_{L^{\infty}}$.

\section{Concluding Remarks}

\subsection{A nonautonomous gradient flow}

As a possible application of Theorem 5.3, one can prove convergence results for the Cauchy problem for the non-autonomous gradient flow:

$$
\dot{x}(t)=-\nabla u(t, x(t)), t>0, x(0)=x_{0}
$$

where $x_{0} \in \mathbb{R}^{d}$ is an arbitrary initial position. Thanks to Proposition 4.2, the previous Cauchy problem possesses a unique solution that is defined for all positive times. An easy consequence of Theorem 5.3 then reads:

Theorem 6.1. Let $x_{0} \in \mathbb{R}^{d}$, and let $x($.$) be the solution of the Cauchy problem (6.1), then x(t)$ converges as $t \rightarrow \infty$ to some point $y_{\infty}$ that is a (global) minimum of $u_{0}^{* *}$.

Proof. Let us denote by $F$ the (convex and compact) set where $u_{0}^{* *}$ attains its minimum. Let $y \in F$, since $\nabla u_{0}^{* *}(y)=0$, using the convexity of $u_{0}^{* *}$ and (5.5), we get

$$
\begin{aligned}
\frac{\mathrm{d}}{\mathrm{d} t}\left(\frac{1}{2}|x(t)-y|^{2}\right) & =\left\langle\nabla u_{0}^{* *}(y)-\nabla u_{0}^{* *}(x(t)), x(t)-y\right\rangle+\left\langle\nabla u_{0}^{* *}(x(t))-\nabla u(t, x(t)), x(t)-y\right\rangle \\
& \leq C \mathrm{e}^{-\lambda t}|x(t)-y| .
\end{aligned}
$$

From which we easily deduce that $|x(t)-y|+\frac{C}{\lambda} \mathrm{e}^{-\lambda t}$ is nonincreasing for every $y \in F$ so that $\min _{y \in F} \mid x(t)-$ $y \mid+\frac{C}{\lambda} \mathrm{e}^{-\lambda t}$ is also nonincreasing. There exists therefore some $d_{\infty} \geq 0$ such that

$$
d(x(t), F):=\min _{y \in F}|x(t)-y| \rightarrow d_{\infty} \text { as } t \rightarrow \infty .
$$

Now we claim that $d_{\infty}=0$; assume on the contrary that $d_{\infty}>0$ and let $y \in F$, we then have

$$
\delta:=\min \left\{\left\langle\nabla u_{0}^{* *}(x)-\nabla u_{0}^{* *}(y), x-y\right\rangle: d(x, F)=d_{\infty}\right\}>0
$$

so that by the same computations as above, we obtain that for large enough $t$, one has

$$
\frac{\mathrm{d}}{\mathrm{d} t}\left(\frac{1}{2}|x(t)-y|^{2}\right) \leq-\frac{\delta}{2}
$$


which contradicts the convergence of $|x(t)-y|$ as $t \rightarrow+\infty$. We thus have proved that $d(x(t), F) \rightarrow 0$ as $t \rightarrow+\infty$ so that all limit points of the trajectory $x($.$) belong to F$. Let $y_{1}=\lim _{n} x\left(t_{n}\right)$ and $y_{2}=\lim _{n} x\left(s_{n}\right)$ with $t_{n}, s_{n} \rightarrow \infty$ be two such limit points, since $\left|x(t)-y_{i}\right|$ converges as $t \rightarrow \infty$ for $i=1,2$, we deduce that $\left|y_{1}-y_{2}\right|=\lim _{n}\left|x\left(t_{n}\right)-y_{2}\right|=\lim _{n}\left|x\left(s_{n}\right)-y_{2}\right|=0$. Together with the compactness of $F$, this proves that $x(t)$ converges to some $y_{\infty} \in F$ as $t \rightarrow \infty$.

\subsection{Interpolation with a geometric flow}

This final section is devoted to an informal discussion on a natural interpolation between the HJB equation (2.1) and some geometric flow. Let us indeed remark that the HJB equation

$$
\partial_{t} u=\min \left(0, \lambda_{1}\left(D^{2} u\right)\right),\left.u\right|_{t=0}=u_{0}
$$

can be imbedded into the family of PDE depending on a parameter $\theta \geq 0$ :

$$
\partial_{t} v=\min \left(0, \lambda_{1}\left(D^{2} v+\theta \nabla v \otimes \nabla v\right)\right),\left.v\right|_{t=0}=v_{0} .
$$

Now, let us formally remark that for $\theta>0, v$ solves (6.3) if and only if $u:=\mathrm{e}^{\theta v}$ solves (6.2) with initial condition $u_{0}:=\mathrm{e}^{\theta v_{0}}$ and therefore admits the representation formula:

$$
v(t, x)=\frac{1}{\theta} \log \left(\inf _{\sigma:|\sigma| \leq 1} \mathbb{E}\left(\exp \left(\theta v_{0}\left(x+\int_{0}^{t} \sqrt{2} \sigma_{s} \mathrm{~d} W_{s}\right)\right)\right)\right)
$$

and, as $t \rightarrow \infty, \mathrm{e}^{\theta v(t, .)}$ converges to the convex envelope of $\mathrm{e}^{\theta v_{0}}$. Letting $\theta$ tend to $+\infty$ in (6.3), we formally obtain:

$$
\partial_{t} v=\min \left(0, \lambda_{1}\left(D^{2} v_{\left.\right|_{\nabla v} \perp}\right)\right),\left.v\right|_{t=0}=v_{0}
$$

which is a geometric flow of motion by the principal negative curvature. One of course expects that the evolution along this flow will tend to convexify the level-sets of $v_{0}$. It is therefore natural to view (6.5) as a quasi-convexification equation. Equation (6.3) therefore interpolates between a convexifying and a quasiconvexifying evolution. There is also a stochastic representation formula for (6.5) due to Soner and Touzi [10], which relies on a stochastic target problem different in nature from (6.4).

Acknowledgements. G.C. wishes to thank the support of the ANR through the projects ANR-07-BLAN-0235 OTARIE and ANR-09-JCJC-0096-01 EVAMEF. A.G. gratefully acknowledges support from Chaire EDF-Calyon Finance and Développement Durable and FiME, Laboratoire de Finance des Marchés de l'Énergie (www.fime-lab.org), and Chaire Axa Assurance et Risques Majeurs. Both authors are grateful to an anonymous referee for several suggestions that led to an improved presentation.

\section{REFERENCES}

[1] O. Alvarez, J.-M. Lasry and P.-L. Lions, Convex viscosity solutions and state constraints. J. Math. Pures Appl. 76 (1997) 265-288.

[2] L. Caffarelli and X. Cabré, Fully nonlinear elliptic equations, American Mathematical Society Colloquium Publications 43. American Mathematical Society, Providence, RI (1995).

[3] Y.G. Chen, Y. Giga and S. Goto, Uniqueness and existence of viscosity solutions of generalized mean curvature flow equations. J. Differential Geom. 33 (1991) 749-786. 
[4] M.G. Crandall, H. Ishii and P.-L. Lions, User's guide to viscosity solutions of second order partial differential equations. Bull. Amer. Math. Soc. 27 (1992) 1-67.

[5] W.H. Fleming and H.M. Soner, Controlled Markov Processes and Viscosity Solutions, Graduate Studies in Mathematics 58. Applications of Mathematics, Springer-Verlag (1993).

[6] B. Kirchheim and J. Kristensen, Differentiability of convex envelopes. C. R. Acad. Sci. Paris Sér. I Math. 333 (2001) $725-728$.

[7] A. Oberman, The convex envelope is the solution of a nonlinear obstacle problem. Proc. Amer. Math. Soc. 135 (2007) 16891694.

[8] A. Oberman, Computing the convex envelope using a nonlinear partial differential equation. Math. Mod. Methods Appl. Sci. 18 (2008) 759-780.

[9] A. Oberman and L. Silvestre, The Dirichlet Problem for the Convex Envelope. Trans. Amer. Math. Soc. (to appear).

[10] H.M. Soner and N. Touzi, Stochastic representation of mean curvature type geometric flows. Ann. Probab. 31 (2003) 1145-1165.

[11] N. Touzi, Stochastic control and application to Finance. Lecture Notes available at http://www.cmap.polytechnique.fr/ touzi/.

[12] L. Vese, A method to convexify functions via curve evolution. Comm. Partial Diff. Eq. 24 (1999) 1573-1591. 